\title{
Infectious discitis caused by Enterobacter cloacae
}

\author{
R Solans, P Simeon, R Cuenca, V Fonollosa, J Bago, M Vilardell
}

\begin{abstract}
The case is reported of a patient who developed a vertebral osteomyelitis caused by Enterobacter cloacae. The organism was isolated in cultures of blood and vertebral puncture biopsy samples. The patient was satisfactorily treated with trimethroprim and sulphamethoxazole.

Enterobacter cloacae, a Gram negative organism, has been confirmed as the cause of bacteriaemia in patients with burns, urinary infections, in adults with pneumonia, and in children with joint infections. Espondylodiscitis caused by Enterobacter cloacae has not previously been described.
\end{abstract}

(Ann Rheum Dis 1992; 51: 906-907)

\section{Case report}

A non-smoking 68 year old woman, who had had pulmonary tuberculosis at the age of 14 years and gallstones with repeated hepatic colic in the three years before this admission, was admitted with a two week history of severe pain in the right hypochondrium that increased with motion, general malaise, anorexia, and dystermia. Physical examination showed fever of $38.7^{\circ} \mathrm{C}$ and tenderness over right hypochondrium. There were no other symptoms such as localised tenderness in the back or lower right ribs.

Laboratory data included: erythrocyte sedimentation rate $127 \mathrm{~mm}$ in the first hour; packed cell volume 0.35 ; haemoglobin $118 \mathrm{~g} / \mathrm{l}$; platelets $282 \times 10^{9} / \mathrm{l}$; and leucocytes $5 \cdot 9 \times 10^{9} / 1$. Urine analysis was normal. One urine culture and five blood cultures were negative. Serology for salmonella and brucella, and bacilloscopy in sputum and urine were also negative. A tuberculin test was negative. A chest radiograph was normal. Radiographs of the spine showed osteoporosis of the dorsal vertebral bodies. Echocardiographs, intravenous urography, and a barium enema were all normal. Following the barium enema, the patient had an acute febrile episode and Enterobacter cloacae resistant to ampicillin and cephalosporins was isolated in blood cultures. A colonoscopic examination was normal. Abdominal echography confirmed the gallstones; no signs of cholecystitis were observed. Bone scintigraphy with technetium99 diphosphonate performed four weeks after admission showed an increase of the isotope in vertebral discs D7-D9. At this time, a spinal column radiograph showed destruction of vertebral disc D8-D9 (fig) which was confirmed by a computed tomography scan.

The presence of an infectious process affect- ing the D8-D9 intervertebral disc was suspected and a puncture biopsy sample was taken at this level which yielded a purulent fluid from which $E$. cloacae were isolated which were ampicillin and cephalosporin resistant and sensitive to gentamicin and trimethroprium with sulphamethoxazole. Trimethroprim and sulphamethoxazole were given intravenously at doses of 160 and $800 \mathrm{mg}$ respectively every 12 hours for 10 days and then treatment continued by mouth. The patient remained in complete repose for four weeks and was discharged without pain and in good health 11 weeks after admission. Repeat radiographs of the dorsal spine performed at four months after admission showed interbody fusion with no signs of activity. The erythrocyte sedimentation rate was $12 \mathrm{~mm}$ in the first hour. Antibiotic treatment was maintained for six months with no complications.

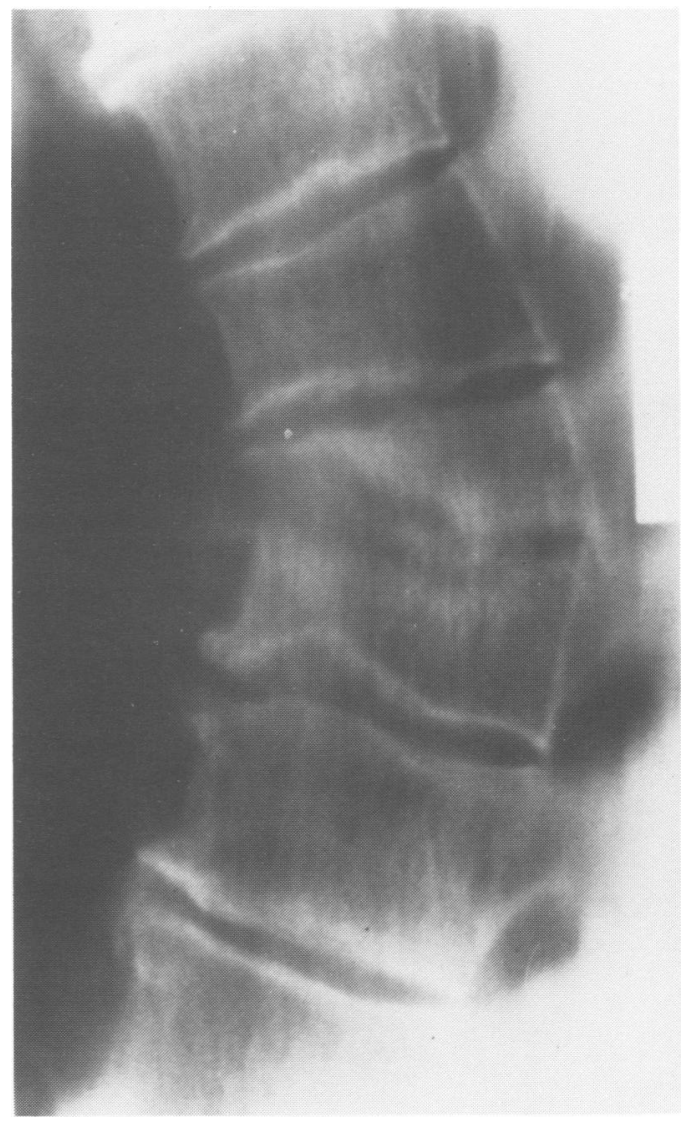

Radiograph of the spinal column showing destruction of vertebral disc $108-1) 9$. 


\section{Discussion}

Vertebral pyogenic osteomyelitis is a rare infection and constitutes only $2-4 \%$ of all cases of pyogenic osteomyelitis. ${ }^{1}$ Its incidence has increased $^{2} 3$ as a result of the greater prevalence of sepsis in patients admitted to hospital arising principally from the urinary tract and intravenous catheters, and the rise in drug addiction. ${ }^{+6}$ The most common causative agent is Staphylococcus aureus, ${ }^{3} 7$ followed by Escherichia colli, ${ }^{48}$ and other Enterobacteriaciae. ${ }^{67}$ Blood cultures are positive in $25-30 \%$ of cases, ${ }^{136}$ and vertebral puncture biopsy allows diagnosis in $50 \%$ of cases. ${ }^{3}$ When the disease affects the lower region of the thoracic spine, referred abdominal pain is common and tends to lead to an erroneous diagnosis. ${ }^{9}$ Diagnosis of pyogenic osteomyelitis of the spine in its initial stage may be difficult due to the lack of clinical specificity. This delay in diagnosis and consequent treatment can lead to severe neurological sequelae. Diagnosis is by radiological studies and isolation of the causative agent from blood cultures or from the infected area by taking a needle biopsy sample. Most patients recover within one year of the onset of illness with adequate antibiotic treatment.

Enterobacter cloacae is a motile, Gram negative bacillus. Enterobacter cloacae and $E$. aerógenes are clinically the two most important species of the genus enterobacter. The enterobacter genus currently accounts for $4-12 \%$ of all cases of Gram negative bacteriaemia. ${ }^{10}$

Enterobacter cloacae has been described as the cause of bacteriaemia in patients with burns, urinary infections, adults with pneumonia, ${ }^{10}$ and children with joint infections. ${ }^{11}$ In 1973 the first case of osteomyelitis of the femur and arthritis of the knee caused by $E$. cloacae in an adult was reported. ${ }^{12} \mathrm{~A}$ case of vertebral osteomyelitis caused by $E$. aglomerans ${ }^{5}$ and four cases due to non-specific enterobacter ${ }^{8}$ have also been described. Enterobacter cloacae is found in the gastrointestinal tract in $2-3 \%$ of patients admitted to hospital and also in the throats of alcoholics and patients who are seriously ill. ${ }^{10}$ Enterobacter cloacae has rarely been isolated from bile (Bourgault et al reported a case of bacteriaemia secondary to biliary tract infection $^{13}$ ), hepatic abscesses, and peritoneal fluid associated with infection after operations. In our patient it was difficult to determine the origin of $E$. cloacae infection. The pathogenic role of this bacterium was well established, however, as it was recovered not only from blood cultures but also from the disc puncture biopsy sample. The colon can be suggested as the source for the enterobacter bacteriaemia for two reasons. First, after the barium enema the patient had an acute febrile episode and $E$. cloacae was isolated in blood culture though no colonic disease was confirmed. Secondly, clinical findings and abdominal echography do not suggest the biliary tract as the origin of infection. Likewise, we believe that $E$. cloacae infection was present before the barium enema bacteriaemia due to the long time between the onset of symptoms and this radiological procedure.
Enterobacter cloacae is generally resistant to ampicillin and cephalosporin ${ }^{10}$ because of the production of a constitutive cephalosporinase. Some strains resistant to aminoglycosides have also been detected, though these are the most active agents against $E$. cloacae ${ }^{10}$ In our patient, $E$. cloacae was sensitive to gentamicin and to a combination of trimethroprim and sulphamethoxazole. The latter was selected due to its pharmacokinetic properties and was given intravenously for ten days and then for six months by mouth. Although there is no general agreement on the duration of treatment, a minimum of six weeks with continuation of the treatment until the patient is asymptomatic and the erythrocyte sedimentation rate returns to normal levels is recommended. ${ }^{2569} \mathrm{Bed}$ rest is needed to relieve the patient's initial discomfort and prevent deformity ${ }^{1}$; bracing is not recommended when the infection is located in the lower dorsal or lumbar regions. ${ }^{5}$

We believe that the most reliable procedures for the diagnosis of septic discitis are the indirect methods of blood culture and radiology (computed tomography scans, magnetic resonance imaging) and the direct method of disc puncture and biopsy. A scintigraphic study with technetium-99 diphosphonate or gallium, or both, allows earlier detection of osteomyelitic foci and allows spinal infection and degenerative disease to be distinguished.

This is a rare case given the causative agent (E. cloacae) and the excellent response to treatment with trimethroprim and sulphamethoxazole. Vertebral pyogenic osteomyelitis, though a rare infection, should be considered particularly in elderly patients with recent onset backache or when long established symptoms of backache worsen. The presence of osteoporotic crush fractures and spondylosis causing backache, common in elderly people, can delay the diagnosis.

The authors thank Miss Christine O'Hara for her help with the English in this paper.

1 Guerrero A, Parras F, Ezpeleta C, Diaz R. Osteomielitis piogenica vertebral hematógena. Enf Infec y Microbiol Cli piogenica verteb

2 Ania B J, Garcia F, Pereda J M. Espondilodiscitis piógena. Rev Clin Esp 1980; 159: 268-73.

3 Griffiths H E D, Jones D M. Pyogenic infection of the spine: a review of twenty-eight cases. $\mathcal{F}$ Bone foint Surg $[B r] 1971$ 53: 383 .

4 Frederickson B, Yuan H. Management and outcome of pyogenic vertebral osteomyelitis. Clin Orthop 1978; 131: $160-7$

5 Musher D M, et al. Vertebral osteomyelitis: still a diagnostic pitfall. Arch Intern Med 1976; 136: 105-10.

6 Zapico A, Montgomerie F. Pyogenic vertebral osteomyelitis: report of nine cases and review of the literature. Rev Infect report of nine cases and
Dis 1979; 1: 754-76.

7 Ross D M, Flemming J L. Vertebral body osteomyelitis. Clin Orthop 1978; 118: 190-8.

8 Lopitaux R, Sirot J, Meloux J. Les infections osteoarticulaires a bacilles Gram negatif. Rev Rhum Mal (Osteoartic 1981; 48: $133-40$

9 Collert S. Osteomvelitis of the spine. Acta Orthop Scand 1977 48: $283-90$.

10 John J F. Sharbaugh R J, Bannister E R. Enterobacte cloacae: bacteriemia, epidemiologv and antibiotic resistance. Rev Infect Dis 1988; 4: 13-28.

11 Gordon S L., Maisels $M$ J, Robbins W J. Multiple join infections with Enterobacter cloacae. Clin Orthop 1977: 125: $136-8$.

12 Ianinni P. Hull S, Quintillani R. Severe sepsis from enterobacter. Arch Surg 1973; 107: 854-6.

13 Bourgault A M, England D M, Rosenblatt J E, Forgacs P, Bieger C. Clinical characteristics of an aerobic bactibilia. Arch Intern Med 1979; 139: 1346-9. 